\title{
BMJ Open Oral medicine modification for older adults: a qualitative study of nurses
}

\author{
Aoife Mc Gillicuddy, ${ }^{1}$ Abina M Crean, ${ }^{2}$ Maria Kelly, ${ }^{1}$ Laura Sahm ${ }^{1,3}$
}

To cite: Mc Gillicuddy A, Crean AM, Kelly M, et al. Oral medicine modification for older adults: a qualitative study of nurses. BMJ Open 2017;7:e018151. doi:10.1136/ bmjopen-2017-018151

- Prepublication history for this paper is available online. To view these files, please visit the journal online (http://dx.doi. org/10.1136/bmjopen-2017018151).

Received 9 June 2017 Revised 27 September 2017 Accepted 16 October 2017

\section{CrossMark}

${ }^{1}$ Pharmaceutical Care Research Group, School of Pharmacy, University College Cork National University of Ireland, Cork, Republic of Ireland

${ }^{2}$ Synthesis and Solid State Pharmaceutical Centre, School of Pharmacy, University College Cork, Cork, Republic of Ireland

${ }^{3}$ Pharmacy Department, Mercy University Hospital, Cork, Republic of Ireland

Correspondence to Aoife Mc Gillicuddy; a.mcgillicuddy@umail.ucc.ie

\begin{abstract}
Objective Oral medicines are frequently modified (eg, tablets crushed) for older adults. However, these modifications can have clinical, legal and/or ethical implications. Nurses bear responsibility for medicine administration and hence, perform these modifications. The aim of this study was to investigate the knowledge, attitudes and beliefs of nurses about oral medicine modification for older adults.

Design A qualitative study was conducted using semi-structured, face-to-face interviews with nurses providing care to older adults in acute and long-term care settings. Interviews were audio-recorded, transcribed verbatim and analysed thematically.

Settings Sixteen purposively selected care settings; 4 acute-care and 12 long-term care settings were included. Nurses were recruited by convenience sampling at these sites.
\end{abstract}

Participants Eighteen nurses participated (83\% female, $67 \%$ long-term care, $33 \%$ acute-care, median age (IQR) 38 years (32.5-52.0)).

Results Three major themes: modifying - a necessary evil, nurses' role as patient advocate and modifying — we are working very much as a team and two minor themes: fractional dosing, and covert administration emerged from the data. Nurses viewed oral medicine modifications as being a routine and necessary occurrence in geriatric patient care due to limitations of available formulations and the presence of age-related challenges in drug administration. Nurses' knowledge of residents' requirements ensured that they advocate for those with individualised formulation needs, however, nurses rely on pharmacists for information about modifications. Nurses expressed a desire for supports including increased education and ward-specific, pharmacist-developed recommendations on common modifications.

Conclusions This study has provided useful insights into the views of nurses regarding oral medicine modification for older adults. The unique and varied formulation requirements of older adults must be acknowledged. Increased engagement by healthcare professionals, the pharmaceutical industry, regulatory agencies and policy-makers is required to facilitate the development of age-appropriate formulations. In the interim, practical interventions, informed by the findings of this study, are required.

\section{INTRODUCTION}

Medication administration is guided by a number of principles, with the ultimate
Strengths and limitations of this study

- The aim of this study was to use qualitative methodology to gain a greater understanding of the knowledge, attitudes and beliefs of nurses about the modification of oral medicines for older adults.

- Thematic analysis, using an inductive approach to coding, facilitated the generation of rich, in-depth, detailed accounts of nurses' views in which the voice of the nurse was given primacy.

- This study was unique as it provided a greater insight into the range of modifications encountered in geriatric care than has previously been reported due to the inclusion of both acute and long-term care settings and the investigation of modifications to overcome swallowing difficulties and to meet patient's dosing requirements.

- The study was conducted in the Munster province, in the south of the Republic of Ireland, with a population of almost 1.3 million. This may limit the transferability to other countries; however, the findings would be transferable to other countries with similar medication licensing and reimbursement procedures.

aim of ensuring that the right medication is administered to the right patient at the right dosage, in the right form and at the right time. ${ }^{12}$ Given the global trend towards an ageing population, ${ }^{3-5}$ combined with the high rates of medication use among the older cohort, ${ }^{67}$ there is widespread recognition of the need to optimise medication use for older adults. ${ }^{8}$ However, various age-related changes, including dysphagia ${ }^{10-12}$ and altered pharmacokinetics (rate and extent of drug absorption, distribution, metabolism and excretion) and pharmacodynamics (the action/effect of a drug on the body), ${ }^{13} 14$ can complicate the administration of the right form or the right dose of oral medication to older adults. This may result in oral dosage forms (ODFs) being modified (eg, tablets crushed or split or capsules opened) to facilitate administration to patients with difficulty swallowing or to tailor the dose to the patient's requirements. Quantitative studies investigating medicine modification for 
older adults have highlighted the prevalence of this practice, as $18 \%$ of all residents in two Australian aged care facilities $(n=160)$ received crushed tablets, ${ }^{15}$ while in a French study, completed in all geriatric units of a university hospital, of the 683 patients included in the study, $32 \%$ received crushed tablets or opened capsules. ${ }^{16}$ Data on tablet splitting for geriatric populations are limited; however, a Canadian study involving 370 nursing home residents found that $35 \%$ of residents received at least one split medication. ${ }^{17} \mathrm{~A}$ study undertaken in an Irish nursing home setting that investigated any modification of an ODF for 111 residents found that $35 \%$ of residents received at least one modified medication. ${ }^{18}$ Modifications have also been widely reported in the literature as being undertaken to facilitate covert administration of medication. ${ }^{19} 20$ This practice has recently been the focus of many policy documents in healthcare given the legal and ethical implications of covert administration. ${ }^{121}{ }^{22}$ Covert administration is not the primary focus of this study, rather the study aims to investigate modifications of oral medicines to meet the needs of older patients in general.

Modifications of ODFs are of concern for healthcare professionals as they can potentially affect therapeutic outcomes for patients and adverse events have been reported as a consequence of medicine modification. ${ }^{23} 24$ There are numerous potential mechanisms by which ODF modifications may alter drug action and therefore, therapeutic outcomes. Administration of a portion of a dosage form, for example, half a tablet, may result in inaccurate dosing. ${ }^{25}$ Modifications, for example, crushing tablets and opening capsules, may alter the site, rate or extent of drug absorption in vivo, which could result in either lower drug levels and lack of efficacy or alternatively, an increase in drug levels and associated toxicity. ${ }^{26}{ }^{27}$ From a regulatory viewpoint, modifications generally void the product licence which can have significant implications for healthcare professionals in the event of adverse events. ${ }^{1}{ }^{28}$ Nonetheless, modifications of oral medicines are prevalent among older patients. ${ }^{15} 171829$ Qualitative study methodologies can be used to gain a deeper understanding of the factors that influence this practice in healthcare.$^{30}$ A number of qualitative studies have investigated medicine modification ${ }^{31-33}$; however, a recent systematic review of qualitative literature highlighted the need for further research in this area given the limited number of studies. ${ }^{34}$ 'The activities associated with medication management involve the nursing, midwifery, medicine and pharmacy professions and the patient/service user', ${ }^{1}$ however, ultimately nurses bear responsibility for medicine administration and therefore, perform modifications and administer modified medicines, in acute and long-term care settings. Despite this, only two previous qualitative studies investigated nurses' views about medicine modification: an interview study conducted with nurses working in nursing home settings ${ }^{33}$; and a focus group study, which investigated the experiences of healthcare professionals (including five nurses) of the challenges encountered in medicine administration to patients with dysphagia. ${ }^{31}{ }^{33}$ Investigation of the views and experiences of nurses about ODF modification will enable a greater understanding of the factors influencing this practice and the challenges encountered by nurses and patients on a daily basis. Increased understanding of these factors will aid the identification of potential areas for prioritisation for intervention and further research. This study contributes to, and further develops, the evidence base in this area, given the inclusion of nurses working in acute and long-term care and investigation of all types of ODF modifications, including fractional dosing.

The aim of this study was to examine the knowledge, attitudes and beliefs of nurses about the modification of ODFs for older adults (aged $\geq 65$ years).

\section{METHODS}

\section{Study design}

Semi-structured interviews were conducted with nurses who provide care for, and administer medicines to, older adults (aged $\geq 65$ years) within the Munster province in the south of the Republic of Ireland.$^{35}$ Semi-structured interviews were chosen as the data generation method as rich, in-depth, detailed accounts of participant's experiences, perspectives and opinions can be gained. In addition, one-to-one interviews are particularly suited for discussing sensitive issues, such as individual nurse's medicine administration practices. ${ }^{30}$

\section{Study setting and sampling}

Purposive sampling, which involves actively selecting the most productive sample to answer the research questions,${ }^{36}$ was used for site identification. A sampling matrix of important variables was developed to ensure that the range of sites providing care for older patients were included. Long-term care settings from each category (public, private and voluntary (publicly funded but governed by a religious or charitable organisation) $)^{37}$; both with and without specialist dementia units were actively sampled. ${ }^{38}$ Previous research has shown that ODF modifications are more common in high dependency units. ${ }^{29}$ According to the Department of Health, the public nursing home sector in Ireland has the highest proportion of maximum dependent older people at just over $60 \%$, compared with private nursing homes where almost $35 \%$ of residents are maximally dependent. ${ }^{39}$ Therefore, the funding category of the long-term care site was used as a surrogate descriptor for dependency. In addition, ODF modifications have been shown to be more common in dementia care units, ${ }^{15}$ providing the rationale for this variable. In the acute-care setting, both acute geriatric hospital wards and acute stroke wards were sought as previous studies have demonstrated that modifications are commonly undertaken in wards of these types. ${ }^{40}{ }^{41}$ Interview participants were identified by convenience sampling of nurses within the purposively selected sites. Where possible, two of each type of care site, and at least one nurse from each site were sought for inclusion 
in the study. For one category of long-term care site, only one such facility was available in the geographical area in which the study was conducted.

The medical director or nurse in charge at each of the purposively identified sites was contacted by telephone or email and provided with details of the study. The person in charge approached individual nurses to identify potential participants. The inclusion criteria for interview participants was any nurse who provides care for, and administers medicines to, older adults (aged $\geq 65$ years) at the purposively selected sites. While healthcare assistants are also commonly employed in acute and longterm care settings in Ireland, their responsibilities centre on personal care and do not usually extend to medication administration. Therefore, in an Irish setting, nurses are responsible for medicine administration, providing the rationale for choosing nurses as the interview participants for this study. It was highlighted to nurses that participation was voluntary and no incentive for participation was offered. When a nurse expressed an interest in participating, the primary researcher (AMG) followed up with a telephone call to arrange a convenient time for the interview.

\section{Data collection}

The topic guide was developed by the authors based on a review of the literature, ${ }^{34}$ observations from a prevalence study ${ }^{18}$ and the authors' practical knowledge of the research area. The topic guide was modified following piloting with an experienced geriatric nurse who provided feedback on the content and language. This pilot interview was not included in the analysis. The topic guide underwent iterative revision throughout the study, to ensure that emerging themes were captured in subsequent interviews. Box provides a summary of the topic guide.

All semi-structured interviews were conducted by AMG, a research pharmacist with training in qualitative research methods and qualitative interviewing techniques. No relationship between the interviewer and participants was established prior to study commencement. The interviews were conducted in a private area at the participant's workplace between March 2016 and February 2017. Only the interviewer and participant were present during the interview. All participants provided written informed consent for participation. Prior to initiating the interview,

\section{Box Summary of topic guide for interviews}

Medicine administration to older adults
Experience of medicine modification
Knowledge about medicine modification
Attitudes and beliefs regarding medicine modification
- Factors influencing practice
Decision-making
- Information sources/resources used
- Healthcare professionals and their involvement
Desire for any further resources/supports

- Medicine administration to older adults

- Experience of medicine modification

- Knowledge about medicine modification

- Attitudes and beliefs regarding medicine modification

- Factors influencing practice

Decision-making

- Information sources/resources used

Desire for any further resources/supports participants completed a demographic data collection form which recorded details including participant's gender, age, qualifications, length of time working with older patients and details of any specific training undertaken in medicine administration. The interviews were audio-taped and transcribed verbatim by AMG. Transcripts were not returned to participants for comment and repeat interviews were not conducted. The interviewer recorded any relevant field notes after conducting the interview.

The method used by Francis $e t a l^{42}$ was used to determine when data saturation had been reached. An initial analysis sample of 15 and a stopping criterion of three were specified a priori. The initial analysis sample was determined based on the prespecified stratification factors in the sampling matrix, which sought to recruit at least one nurse from each purposively selected study site. The stopping criterion of three required that a further three consecutive interviews were conducted, in which no new concepts emerged, to confirm that data saturation had been achieved.

\section{Analysis}

The 'Thematic Analysis' approach, as described by Braun and Clarke,${ }^{43}$ was used to analyse the data. The data (transcripts) were inputted into QSR International's NVivo V.10 Qualitative Data Analysis Software to facilitate analysis. Thematic analysis involves six phases: familiarisation with the data, generation of initial codes, searching for themes, reviewing themes, defining and naming themes and producing the report. Data familiarisation began at an early stage with data transcription, reading and rereading of the data. Open coding (phase II) was undertaken by one author (AMG) to generate initial, non-hierarchical codes. These initial codes were then categorised and re-ordered to generate potential themes. The next step involved reviewing and refining the themes generated in phase III. The fifth stage involved further analysis to refine the themes and to generate clear definitions and names for each theme. The final stage involved drafting the report. Participants were not asked to provide feedback on the study findings.

To ensure that codes were applied consistently, a coauthor (MK) independently coded a random sample of three interview transcripts. The inter-rater reliability between coders was determined by calculating the kappa coefficient for interviews coded by AMG and MK. The kappa coefficient measures the level of agreement, and ranges from 0 to 1 ; with 1 indicating perfect agreement and 0 indicating no agreement. ${ }^{44}$ In addition, each of the coauthors (LJS, AMC and MK) read six interview transcripts to assess if the themes were reflective of the interview content, to further ensure the confirmability of the findings.

\section{Reflexivity}

The research team sought to address reflexivity during the design of the study. All four authors are pharmacists, 
and all are female. Two are academic staff members (one in clinical practice and one in pharmaceutics) and at the time of the study, the other two researchers were $\mathrm{PhD}$ students (both in clinical practice). None of the researchers was employed at any of the study sites and they had no prior relationship with any of the individual nurses who participated in the study. All members of the research team have been involved in research investigating medicine modification for older adults. The research team discussed their preconceptions and thoughts about the research area and all felt, based on their previous experience, that medicine modification was likely to be encountered by nurses in geriatric patient care. However, all the research team acknowledged that they were unaware of the challenges encountered when physically performing medicine administration and modification as none had any previous practical experience in this area. Therefore, the views of nurses were given primacy and an inductive approach was seen to be most appropriate.

\section{Reporting}

This study is reported in accordance with the Consolidated Criteria for Reporting Qualitative Research (COREQ) guidelines. $^{45}$

\section{RESULTS}

\section{Characteristics of interview participants}

Eighteen interviews were conducted in total. The interviews ranged in length from 7 to $31 \mathrm{~min}$, with a mean interview duration (SD) of $16 \mathrm{~min}(6 \mathrm{~min})$. Twelve of the interviewed nurses worked in long-term care and six worked in acute care. Of the nurses who participated in the study, $83 \%$ were female. The median age of participants was 38 years (IQR 32.5-52.0). Seventeen nurses provided details about their experience caring for older people and the median length of experience in geriatric nursing was 8 years (IQR 5.0-11.5). Table 1 describes the characteristics of the interview participants.

\section{Intercoder reliability}

A Cohen's kappa score of 0.924 was obtained which demonstrated aligned thinking between coders. In addition, following review of the themes in relation to interview content, all authors agreed that the themes generated were representative of the content of the interviews.

\section{Themes}

Three major themes emerged from the data: modifying-a necessary evil, nurses' role as patient advocate, modifying - we are working very much as a team. In addition, two minor themes emerged: covert administration, and fractional dosing. In order to comprehensively discuss nurses' views on the topic of medicine modification, these two minor themes will be briefly addressed.
Table 1 Characteristics of interview participants $(n=18)$

\begin{tabular}{|c|c|}
\hline Characteristic & $\mathbf{N}(\%)$ \\
\hline \multicolumn{2}{|l|}{ Gender } \\
\hline Male & $3(17 \%)$ \\
\hline Female & $15(83 \%)$ \\
\hline \multicolumn{2}{|l|}{ Age group (years) } \\
\hline 20-29 & $1(6 \%)$ \\
\hline $30-39$ & $10(56 \%)$ \\
\hline $40-49$ & $2(11 \%)$ \\
\hline $50-59$ & $4(22 \%)$ \\
\hline $60-69$ & $1(6 \%)$ \\
\hline \multicolumn{2}{|l|}{ Nurse profession } \\
\hline Staff nurse & $4(22 \%)$ \\
\hline CNM 1 & $1(6 \%)$ \\
\hline CNM 2 & $5(28 \%)$ \\
\hline Assistant Director of Nursing & $1(6 \%)$ \\
\hline Nurse (not specified) & $7(39 \%)$ \\
\hline \multicolumn{2}{|l|}{ Care setting $(n=16)$} \\
\hline Public LTCF with SDU (n=2) & $2(11 \%)$ \\
\hline Public LTCF without SDU (n=2) & $2(11 \%)$ \\
\hline Voluntary LTCF with SDU $(n=1)$ & $1(6 \%)$ \\
\hline Voluntary LTCF without SDU (n=2) & $2(11 \%)$ \\
\hline Private LTCF with SDU $(\mathrm{n}=3)$ & $3(17 \%)$ \\
\hline Private LTCF without SDU $(n=2)$ & $2(11 \%)$ \\
\hline Geriatric ward in acute hospital $(n=2)$ & $4(22 \%)$ \\
\hline Stroke ward in acute hospital $(n=2)$ & $2(11 \%)$ \\
\hline \multicolumn{2}{|l|}{ Medicine administration training completed } \\
\hline Yes & $17(94 \%)$ \\
\hline No & $1(6 \%)$ \\
\hline
\end{tabular}

Medication administration training mentioned included: on-site medication management training courses, refresher courses, online training, undergraduate training, pharmacy-provided training. CNM, Clinical Nurse Manager; LTCF, long-term care facility; SDU, specialist dementia unit.

\section{Major themes \\ Modifying-a necessary evil}

Modifications of oral medicines were viewed by participants as being a routine and necessary part of clinical practice and were undertaken on a daily basis as part of drug rounds. It was strongly felt that the older cohort in particular require modified medicines more frequently, "To be honest I think that modifying medicines is a necessity, especially in elderly patients" (Nurse 2, Long Term Care (LTC)), "It would be very common here in the unit. It's a geriatric ward. it would be very common I suppose here because of our patient group" (Nurse 10, Acute Care (AC)). Participants discussed a number of different types of modifications that they encountered, including tablet crushing, capsule opening, tablet splitting, dispersing or dissolving tablets and mixing medications with food. However, the modification that was reported as being 
most common was tablet crushing, "In $95 \%$ of cases when we are talking about modifying medicines we are talking about crushing" (Nurse 5, LTC). The necessity to modify medications was seen to be an inevitable part of older patient care and participants highlighted common reasons for this including: swallowing difficulties or dysphagia, medical conditions with dysphagia as a sequela, patient preference or difficulty with large dosage forms, family input and formulation characteristics. Overall, age-related swallowing difficulties and dementia were the most commonly implicated reasons for ODF modification. It was clear from the interviews that formulation suitability is extremely individualised, "all patients are so different... so you'd be looking at lots of different types of medications... whatever fits in with the individual patient" (Nurse 9, AC) and it is vital that each patient's needs are assessed on a regular basis due to the potential for fluctuations in formulation suitability,

...you can see progressively the swallow or the level of cognition fluctuates, that affects the swallow and it's something that you are looking at and thinking, oh my god (sic), this patient actually was taking oral tablets a matter of weeks ago and now it's a case of that we're dispersing them and giving them different suspensions... we are just keeping an eye out for ourselves [nurses watch for changes in patient's ability to take different mediciation formulations] (Nurse 9, $\mathrm{AC)}$.

Participants reported that alternative formulation options are investigated when patients experience difficulty with solid ODFs. Options discussed included changing the route of administration or the formulation of the medication for example, liquid formulations, transdermal patches, dispersible tablets, etc. The availability of appropriate alternative formulations was satisfactory for certain classes of medications, for example, antibiotics and antidementia medications; and participants expressed that in many instances the use of these alternatives was preferable to modifying oral medicines. However, there was an almost universal acknowledgement that there were significant limitations associated with alternatives that often resulted in modifications of ODFs being required or even preferred including: lack of availability or difficulty sourcing alternatives; cost; alternatives not being covered on reimbursement schemes; difficulties administering large or small volumes and issues with the viscosity of the liquids that may increase the risk of aspiration, “... then we have liquid forms, again it's hard to give liquid form medications to people with swallowing difficulties because of aspiration" (Nurse 13, LTC).

Participants expressed a wide variety of attitudes and beliefs about ODF modification. As stated, it was clear that the majority of participants felt that modifications were a routine part of practice. Participants highlighted a number of benefits of modifying ODFs including ensuring that vital medications are administered and overcoming concerns about not modifying medications, for example, the risk of choking or discontinuation of necessary medication, "Your choice is ... crush the medicine which is what we do. or give the medication in its uncrushed form and run the risk of the person choking on it or not give the medicine at all and then, you know, the risk of the illness that's treating" (Nurse 5, LTC). However, notwithstanding the general acceptance of modifications as a routine occurrence, participants reported numerous concerns about modifications including: inaccurate dosing, altered drug absorption or effectiveness, potential interactions with food vehicles used for administration, possible cross-contamination issues, occupational hazards for nurses due to exposure to powdered drugs and the unlicensed nature of the modified medicines,

Sometimes I question it because... you crush all the tablets, they are all kind of going into one, you know, one dust...so are you modifying you know... the chemistry or what the tablet actually does because each tablet is made up individually... Plus, I don't know are they getting the full dose of what they should be getting, because obviously there is going to be residue inside in the crush pouch that you can't ever get out, and also... some in the yoghurt, or in the yoghurt tin or whatever you mix it in. So am I'd say they probably don't always get the right dose (Nurse 13, LTC).

An additional issue identified by nurses was the time-consuming nature of modifications, which impacted on nurses' time and work-load, on the patient receiving the modified medicine and on other patients in the ward, "It definitely affects nurse's time...we have a resident here ... it takes twenty minutes to finish only that patient because everything needs to be crushed individually and you have to give everything individually. So it takes a good bit of time from you...that will affect the other patients as well... time is everything" (Nurse 1, LTC).

Overall, it is clear that nurses view modifying ODFs as a key part of their medication administration role. While nurses are aware that modifications may not be appropriate and did express concerns about the effect of modifications on drug action, it is accepted that to meet the many individualised and varied needs of older patients, medicine modifications are a necessity, "Look it's essential....it's a necessary evil... I'm all for modifications because it's necessary" (Nurse 4, LTC)

\section{Nurses' role as patient advocate}

Medicine administration was acknowledged by participants as being a key aspect of their role and nurses viewed medicine administration as their area of expertise. However, from the interviews, it was clear that the responsibilities of nurses extended far beyond simply modifying and administering the medication. Nurses are at the frontline of healthcare provision and play a central role in every aspect of patient care related to medicine modification. Nurses: identify when patients are experiencing difficulty swallowing medications; arrange 
review by appropriate healthcare professionals; highlight patient's requirements and needs to other healthcare professionals, especially doctors and pharmacists when prescribing and dispensing and communicate and liaise both with and between members of the multidisciplinary team. Therefore, the input of nursing staff is crucial in the area of medicine modification, "Very much nurse led..." (Nurse 6, LTC). The importance of nurses knowing their patients was central to this, "...one of the huge advantages of working in care of the older person in long term settings is that you really get to know the patient..." (Nurse 7, LTC). Nurses, particularly in long-term care, know the patients, and therefore their preferences and requirements, extremely well, "the most important thing is to know the person, know each individual" (Nurse 8, LTC). Nurses are also alert to subtle changes in the patient that may suggest that the patient is experiencing difficulty, “... as their medical condition changes... they've become unwell and we know that, you know, they're coughing a lot or that their swallow has changed or that they're not able for their big medications, it's because we know our patients so well I suppose" (Nurse 17, LTC). Participants did acknowledge that although some healthcare professionals do consider patient's formulation requirements, particularly those who know the patient or geriatric specialists, overall there was a reliance on nurses to communicate patient's formulation requirements. Nurses were cognisant of this responsibility and were particularly aware of highlighting this to out-of-hours doctors, "Especially [out-of-hours GP service] you have to be there on top of them saying no, this person is liquid, this person is, you know, suspension or whatever" (Nurse 6, LTC) and locum nursing staff. Nurses also shoulder responsibility for liaising between the various members of the multidisciplinary team and communicating various recommendations within the team as often the multidisciplinary team members communicate through the nursing staff rather than directly, "The pharmacist will do a review, the pharmacist leaves instructions, I communicate them to the GP" (Nurse 17, LTC).

It was evident that care provision in the area of medicine modification is very much nurse-led and nurses play a central role in this area. Nurses appeared to be confident in exercising these responsibilities and at the centre of this was their acknowledgement that knowing the patient and developing a good relationship with them is a key component of their role. This allows nurses to act as an advocate for their patients. For nurses, acting as an advocate for their patient's, is an intrinsic component of their professional responsibility and identity "...none of them can speak for themselves, so you have to have somebody that knows them to be able to" (Nurse 8, LTC).

\section{Modifying - we are working very much as a team}

Nurses believed that decision-making around medicine modification requires the input and expertise of many different members of the multidisciplinary team. Nurses repeatedly highlighted the importance of ensuring that modifications are safe and appropriate "So we'd always make sure that if we're crushing tablets that it's safe to do so" (Nurse 18, AC). Nurses throughout the interviews demonstrated knowledge that certain formulations should never be modified, for example, sustained-release and enteric-coated preparations, which was based on their previous experience with such medications, "We'd know as well... the slow release, long acting, enteric coated or retard medicines... that we couldn't be crushing them" (Nurse 14, LTC). Ultimately however, nurses acknowledged that drug formulations and modification appropriateness was not their area of expertise, "We would always seek external advice for that because that's not our area of expertise" (Nurse 7, LTC), and that they always sought information and advice prior to modifying oral medicines, "I always say it, I'm not a pharmacist and I'm not a doctor and I think it's something that, I'm very cautious by nature anyways, am so it's something I always double and triple check" (Nurse 9, AC).

The main information source for nurses about the modification of oral medicines and the availability of alternative formulations was the pharmacist, "It is always the pharmacy" (Nurse 7, LTC). Nurses reported relying on information provided by the pharmacist, "If I have any concerns I always ring the pharmacist and I always go by their directions...but always check with the pharmacist" (Nurse 2, LTC), "That would be a rule that we just don't go off modifying the tablets ourselves, we have to do it in liaison with the pharmacy department so that we know..... the patient is getting the benefit of the medication, it is not altering the effectiveness of the drug and that it's safe for the patient" (Nurse 10, AC). In general, nurses had very positive views of pharmacists, "Our pharmacists are excellent. They are very accommodating to us" (Nurse 3, LTC) and pharmacists were seen to be the most knowledgeable member of the multidisciplinary team in relation to modifications, "On a Sunday or a bank holiday, you could discuss it with the medical team on call but often... regs [registrars] will tell you really to refer to the pharmacy department as soon as possible because I suppose, they've the most knowledge in relation to medication, the altering and modifying of them" (Nurse 10, AC). One nurse did express dissatisfaction with the support provided by a pharmacy in the past, "There was one pharmacy alright that were a bit slack that I worked with" (Nurse 6, LTC). However, this reliance on pharmacists did present challenges in acute-care settings when the pharmacy department is closed, "We don't have any 24 hour pharmacy... you know so it may not be appropriate forms, it could be whatever you can get" (Nurse $15, \mathrm{AC})$.

Nurses did mention a number of reference sources, for example, British National Formulary, Monthly Index of Medical Specialities (MIMS) and Summary of Product Characteristics; however, these resources did not provide information related to medicine modification "And it's not really in the MIMS either... it doesn't say whether you can crush it or not" (Nurse 16, LTC). In one acute-care 
setting, a ward-specific guideline on medicine modification was developed by the pharmacy department, which was mentioned as a useful resource available at the moment of medication administration, "Just definitely, that do not crush book, that's like our bible here" (Nurse $12, \mathrm{AC})$.

The attitudes of interview participants differed regarding the need for more resources and supports. Many of the participants expressed satisfaction with the supports and systems in place in their workplace. The majority of participants reported that they had very good working relationships with other members of the multidisciplinary team and they valued a collaborative approach to decision making in which their opinions were listened to and accepted, "We have a very good working relationship but generally they don't question, they accept, you know, our judgement on it I suppose" (Nurse 5, LTC). Notwithstanding this finding, some participants did discuss a number of potential methods of improving multidisciplinary collaboration including: the multidisciplinary team meeting to review and discuss patient's needs and greater consideration being given to patient's formulation requirements by doctors and pharmacists when prescribing or dispensing, "I suppose, as a multidisciplinary, sitting down as a nurse, GP and pharmacist together... there isn't enough constructive reviewing of that, it's very much the GP does the monthly round, the script goes to the pharmacist, the pharmacist sends it up and the follow-up really is the nurse" (Nurse 7, LTC). Some participants also expressed a desire for auditing of their medication administration practices, "I suppose observing how we are doing it because again, you can't constructively say right that's the correct procedure unless you actually observe it yourself" (Nurse 7, LTC). A number of other suggestions were mentioned including: more education and training from pharmacists and availability of a pharmacy-developed site-specific formulary or online database on commonly encountered modifications, "I think all nurses should be given extra pharmacy education, especially in specialist areas where we are doing a lot of modifications" (Nurse 9, AC), "A pharmacy-led manual on the ward where, whatever amount of medications they can go through, whether they state whether this medication could be crushed or halved or dissolved in water or whether, you know, it is readily available in that particular hospital in liquid form" (Nurse 10, AC). Nurses also stated that increased availability of alternative formulations and increased recognition by the pharmaceutical industry of the formulation challenges encountered by older patients on a daily basis would be welcome.

\section{Minor themes}

Fractional dosing

Fractional dosing involves the administration of part of a dosage form, for example, half a tablet, to facilitate the administration of a lower dose. While score lines are often present on tablets, in many instances these score lines do not divide the tablet into equal doses but rather allow tablets to be split to facilitate swallowing. When participants were asked to discuss the common modifications they encountered in practice and the reasons for undertaking modifications, none of the participants volunteered fractional dosing. While splitting tablets was commonly mentioned, this was to overcome difficulty swallowing large tablets rather than for fractional dosing. However, when participants were specifically asked about modifications for fractional dosing, it was viewed as being frequently undertaken for older patients, "Yeah, daily ... some of the medication that we give out on a daily basis doesn't come in a dose that's prescribed" (Nurse 11, AC). Fractional dosing was felt to be necessary for older adults due to a combination of increased sensitivity to higher doses and a corresponding lack of commercial formulations that meet these dosing requirements, "I see a lot more of it here [fractional dosing on a geriatric ward] ...you would find that they're constantly altering doses because some of them might be too severe, patients don't react well and it's always finding the fine balance to keep some of the patients on an even keel" (Nurse 9, AC), "...splitting the tablet because the form, the dosage wouldn't be available, especially with the anti-hypertensive tablet, we always split it or quarter it" (Nurse 1, LTC). Drugs acting on the central nervous system, especially quetiapine, and the cardiovascular system were the most commonly implicated medications, "Seroquel ... comes in a $25 \mathrm{mg}$ tablet. That has to be split for 12.5. Yet 12.5 is the most common dose... but there is no 12.5 available" (Nurse 6, LTC). Fractionally dosed medications were often supplied presplit by the pharmacy, which seemed to account for nurses not considering this a modification as they did not physically perform the modification themselves, "The pharmacy will do the alteration if they are required, we don't do it at floor level no" (Nurse 17, LTC). In addition, there was a general belief that scored tablets are designed to be split in two for fractional dosing, "I mean many tablets as you know are scored to be divided in two" (Nurse 5, LTC). However, some nurses did report checking with pharmacy colleagues whether fractional dosing was appropriate, even for scored tablets. While overall participants did not have many concerns about administering fractionally dosed medications, particularly if the pharmacy had split the tablets in advance or the tablets were scored, a number of concerns were raised including inaccurate dosing, wastage and difficulties splitting tablets, "How accurate is the dose?... like, as somebody said to me 'I gave him the big half'. Yeah, that says everything" (Nurse 6, LTC). Overall, participants' attitudes to the modification of medications for fractional dosing were distinct from attitudes to modifications due to swallowing difficulties or patient preference. This appeared to be related to lack of knowledge about the purpose of score lines on tablets and different attitudes towards modifications when tablets are presplit by the pharmacy. 


\section{Covert administration}

The modification of medicines to facilitate covert administration was mentioned by a number of participants and it would be remiss not to address this as a separate theme. A number of participants acknowledged that covert administration was commonly undertaken in the past, particularly for patients with dementia or agitated patients, "Now I would admit, I'm a long time in the profession, certainly that has happened in the past" (Nurse 5, LTG). The majority of participants who discussed covert administration stated that it was not undertaken at their site of employment, "Now, it's not actually done in this hospital" (Nurse 6, LTG) and that it is no longer commonly encountered. The ethical implications of covert administration and the importance of respecting patient's wishes was a major factor influencing this, "Oh no, no, we would never... if a patient refuses that's it... the law as you may know, says no means no. And no even means no from somebody who is cognitively impaired" (Nurse 5, LTC). There was an acknowledgement that very occasionally, covert administration occurs but that this is undertaken in strict adherence to detailed policies and guidelines on covert administration, following discussion with all members of the multidisciplinary team and the patient's family and taking the importance of the medication into consideration, "We have a policy around it and I suppose it would have to be in the person's best interest whether they need the tablet or not" (Nurse 16, LTC). The main challenge discussed by nurses relating to covert administration was the ethical dilemma that arose when trying to balance respecting patient's wishes and providing optimal medical and pharmacological care to individual patients.

\section{DISCUSSION}

This study has identified the knowledge, attitudes and beliefs of nurses about the modification of oral medicines for older adults using qualitative, semi-structured interviews. Three major themes emerged from the data: modifying-a necessary evil; nurses' role as patient advocate; modifying - we are working very much as a team. The findings of this study provide important insights that will enable us to better understand ODF modification practices and the challenges that need to be addressed to optimise formulation suitability for older adults.

From the results of this study, it is clear that nurses view ODF modifications as being a necessity in the care of older patients. While a myriad of factors contributed to this, one of the most important influences was the belief that formulation suitability is extremely individualised and varies significantly between patients due to medical conditions, patient preference, severity of dysphagia and the clinical status of the patient. This finding concurs with a recently published systematic review on the views of healthcare professionals and patients about medicine modification which found that patient-centred individuality and variability was a key driver of ODF modification. ${ }^{34}$ While it was noted that alternatives to solid ODFs are often simply not available, it was also highlighted that modifications are often preferred due to unsatisfactory properties of available alternative oral liquid formulations such as viscosity and the consequent risk of aspiration, expense, difficulties sourcing and difficulties administering the necessary volume. These reported challenging properties of alternative formulations echo the findings of previous qualitative studies. ${ }^{31} 33$ This study further develops the evidence base through the inclusion of nurses working in both acute and long-term care settings and also by investigating modifications to facilitate fractional dosing which have been neglected in qualitative literature thus far. Data from quantitative studies have demonstrated that modifications are prevalent, ${ }^{15} 182946$ and alternative formulations are often unavailable or unsatisfactory, ${ }^{18} 47$ which confirms the beliefs expressed by nurses about modifications. However, despite this reality, guidance provided to healthcare professionals generally advises that modifications should be avoided. ${ }^{127}$ It is clear that this advice can be difficult for healthcare professionals to adhere to given limitations of currently marketed ODFs.

The nurses' role as patient advocate was a strong theme present throughout the data. Familiarity with, and knowledge of, the patient ensures that nurses have a vital role in identifying patients' difficulties with oral formulations and liaising with other healthcare professionals to address any issues. Previous studies have found that inadequate communication practices result in lack of awareness of patient's formulation requirements. ${ }^{34} 48$ This study has found that for patients in long-term and acute-care settings, nurses' knowledge of the patient allows them to act as an advocate on the patient's behalf which helps to overcome this communication deficit. This is particularly true in long-term care given the length of stay. On acute geriatric wards, nurses spend more time on direct nursing care compared with many other ward types ${ }^{49}$ and have more direct patient contact than any other healthcare professional, facilitating the development of the nurse-patient relationship. A key tenet of nursing care and the subject of much nursing literature is the concept of 'knowing the patient'. ${ }^{51}$ The importance of the nurse-patient relationship has been suggested as being an important contributor to the individualisation of care provision and potentially improving patient outcomes. ${ }^{51}{ }^{52}$ This finding has important implications for other members of the multidisciplinary team who should be encouraged to consult with nurses as they 'know' the patient prior to prescribing and dispensing medications for this cohort. A more formal, proactive review of patients, in the presence of all necessary members of the multidisciplinary team, was also mentioned as a possible method to optimise formulation suitability for individual patients in the absence of satisfactory alternatives. An approach such as this would ensure that all necessary expertise was available and would allow other healthcare professionals to benefit from the nurses knowledge of the individual and decrease the time spent by nurses in communicating recommendations from one healthcare 
professional to another. Administrative tasks, such as these, can be time-consuming for nurses which takes time away from direct patient care. ${ }^{53}$ The importance of a patient advocate needs to be recognised by healthcare professionals, which has particular implications for community-dwelling patients. Studies have previously reported that healthcare professionals are frequently unaware of difficulties with medication intake experienced by community-dwelling patients, ${ }^{54-56}$ which highlights the importance of enquiring about difficulties, either from the patient themselves or a suitable advocate who knows the patient, for example, a carer.

Some nurses in this study expressed a desire for resources and supports that would help them in their day-to-day practice. Nurses were reliant on pharmacists for information provision regarding the appropriateness of medicine modification and satisfaction with pharmacists' role was generally expressed. In addition, while not a major source of information, nurses also expressed satisfaction with the role of the doctor in their workplaces, who support nurses in ensuring that modifications are authorised on drug charts. Some nurses did express a desire for further education and training on the pharmacological and pharmaceutical implications of modification, and while they did acknowledge that this was not their area of expertise, some felt that increased knowledge would facilitate nurses to be more empowered in this area. There were variations in nurse knowledge displayed, which may serve as starting points for educational interventions, particularly in the area of capsule opening and fractional dosing. Knowledge about the purpose of the score line on tablets differed, with some nurses assuming that this score line was to facilitate fractional dosing and therefore, divided the tablets into two equal halves. This has been identified as an issue previously ${ }^{57}$ and regulatory agencies are becoming more concerned about the presence of non-functional score lines on tablets. ${ }^{58} 59$ This may highlight a potential role for education of nurses on relevant, fundamental aspects of pharmaceutics (dosage form design). Nurses seemed to express a preference for pharmacists to deliver such educational programmes. Another possible intervention suggested by nurses was the use of a pharmacist-developed, site-specific guidance document, which would provide recommendations on commonly encountered modifications within the care setting. One study site had such a resource and the participant found this to be extremely useful in their daily practice. Bourdenet $e t$ $a l^{t 0}$ found that the implementation of good practice recommendations and the development of a list of medications that should not be modified resulted in a significant reduction in the number of patients receiving crushed medication and a significant reduction in crushing of drugs that should not be modified. While practical interventions such as this should be developed and evaluated in an attempt to support nurses and patients administer medications in the short-term, ultimately there is a need to increase the availability of licensed formulations that meet the needs of the older adult. Nurses advocate for individual patients in daily practice; however, the older cohort requires advocates to highlight their needs to industry, regulatory agencies and policy-makers, to encourage the development of age-appropriate formulations. Optimisation of formulation suitability for older adults will require a thorough understanding of the challenges of administering medication to older adults. The literature abounds with commentaries on the age-related challenges in medication administration. ${ }^{8061}$ A growing body of evidence from clinical practice is required to quantify the breadth of the problems encountered. However, to truly understand the challenges encountered, the views of healthcare professionals, patients and carers need to be heard to ensure that any potential solutions developed reflect the priorities and needs of end-users ${ }^{62}$ and therefore, will be of maximal benefit. ${ }^{62}$ This study adds to the existing evidence-base on the views of nurses about medicine modification. ${ }^{31}{ }^{33}$ In addition to the potential interventions discussed above, further research should investigate the views of patients and their informal carers in community settings where there may be limited healthcare professional input into decision-making in relation to medicine modification. ${ }^{555}$ This research should seek to identify the main challenges encountered by this cohort, and to determine the 'ideal' formulation characteristics from the perspectives of patients, carers and healthcare professionals.

There are a number of strengths associated with this study. The use of semi-structured interviews allowed for in-depth, detailed accounts of participants' experiences and perceptions to be elucidated. ${ }^{30}$ The data were analysed thematically, ${ }^{43}$ using an inductive approach to coding which produced rich findings that were firmly rooted in the data. The timeframe of the study also facilitated an iterative approach to data collection and analysis, which allowed a thorough interrogation of the data. The transferability of the study findings may be questioned given that the interviews were conducted in one geographical area in Ireland. However, the use of the sampling matrix helped to ensure that the views of nurses working in a variety of care settings were elucidated, which helped to overcome this limitation. In addition, the findings cohered with the limited evidence from other qualitative and quantitative studies, which further confirm the transferability of findings. Social desirability is another potential limitation of the study. Participants were aware that the interviewer was a pharmacist by background and the research team were all based at a School of Pharmacy, therefore, there is the possibility that the participants gave socially desirable answers. While it is difficult to eliminate this bias, the research team had discussed this prior to undertaking the study and felt that it would be unethical not to disclose the research team's backgrounds. However, this was balanced by highlighting to participants that the research team were interested in hearing and gaining a greater understanding of the views and experiences of nurses. Social desirability did not appear to emerge as a significant issue given the honest, forthcoming nature of the interviews, with both positive and negative experiences with pharmacy colleagues being reported and 
discussed. In addition, nurses were asked to describe the procedure followed in their institution when medications were required to be modified for patients. Nurses, in different institutions, all described the input of pharmacists and the role they played. The role of the pharmacist was similar across all institutions included in the study, so it is likely that this reflects the true role that pharmacists play in an Irish setting. The length of the interviews ranged from 7 to $31 \mathrm{~min}$. The shortest interview was conducted on an extremely busy ward in an acute hospital, which is likely to have contributed to the brevity of this interview. However, useful insights were still gained in this interview. The interviews focused on oral medicine modification, which was clear to participants from the information leaflet for the study. As a result, the discussions in the interviews centred on medicine administration and modification for older adults from the outset. Therefore, very detailed insights into this topic were gained from what could be considered to be relatively short interviews. The authors feel that the interviews provided comprehensive coverage of the topic and therefore the length of the interviews has not impacted on the quality or depth of findings of the study. It was not possible from the detail provided in the completed data collection forms to ascertain the primary nursing qualification of all participants due to variability in how nurses completed the data collection form. Regardless of qualification, all of the interview participants would be required to demonstrate their competency to, and register with, the Nursing and Midwifery Board of Ireland and adhere to the relevant national guidelines on medicine administration. In addition, the number of years working in care of older patients was recorded, but not the total number of years since qualification, as the former was of more interest for the purposes of this study. No variation in ideas or responses was observed between participants based on age, qualification type or length of experience in geriatric patient care. However, previous research has investigated how nursing competence relates to length of clinical experience. ${ }^{63}$ While in this study, similar themes and insights were gained irrespective of length of experience in geriatric patient care, future research should consider qualification type and time since qualification to investigate whether this affects practice.

\section{CONCLUSION}

This study has provided a useful insight into the knowledge, attitudes and beliefs of nurses on the modification of oral medicines for older adults. Modifications of oral medications are viewed as unavoidable in care of the older person, due to limitations of available formulations. Nurses had a number of concerns about modifications and valued input from other healthcare professionals, particularly pharmacists. Nurses' knowledge of individual patients ensures that nurses have a vital role to play in identifying and assessing patients experiencing difficulty with oral medicines and in communicating with other healthcare professionals. The unique and varied formulation requirements of older adults must be acknowledged by healthcare professionals, academics, regulatory agencies, the pharmaceutical industry and policy-makers to promote the development of more age-appropriate formulations. In the interim, practical interventions and guidance should be developed, taking into consideration the themes that were identified in this study.

Acknowledgements The authors wish to thank all the nurses who participated in the study. In addition, our thanks to all the study sites who facilitated the conduct of this study. The support and guidance of Ms. Gerardine Bowler is gratefully acknowledged.

Contributors AMG was responsible for the conception and design of the study, data collection, data analysis, drafting and critical revision of the manuscript and approval of the final manuscript. AMC and LJS were involved in the conception and design of the study, discussions around data analysis and interpretation, critical revision of the manuscript and final approval of the manuscript. MK was responsible for analysis and interpretation of the data, critical revision of the manuscript and approval of the final manuscript.

Funding AMG is in receipt of a Government of Ireland Postgraduate Scholarship from the Irish Research Council (grant number G0IPG/2016/1634) and was previously in receipt of a University College Cork Strategic Research Fund $\mathrm{PhD}$ Studentship; AMC is a funded researcher in the Synthesis and Solid State Pharmaceutical Centre supported by the Science Foundation of Ireland under grant number $12 / \mathrm{RC} / 2275$.

\section{Competing interests None declared.}

Ethics approval Ethical approval for this study was granted by the Clinical Research Ethics Committee of the Cork Teaching Hospitals, Cork, Ireland.

Provenance and peer review Not commissioned; externally peer reviewed.

Data sharing statement No additional data are available.

Open Access This is an Open Access article distributed in accordance with the Creative Commons Attribution Non Commercial (CC BY-NC 4.0) license, which permits others to distribute, remix, adapt, build upon this work non-commercially, and license their derivative works on different terms, provided the original work is properly cited and the use is non-commercial. See: http://creativecommons.org/ licenses/by-nc/4.0/

(C) Article author(s) (or their employer(s) unless otherwise stated in the text of the article) 2017. All rights reserved. No commercial use is permitted unless otherwise expressly granted.

\section{REFERENCES}

1. An Bord Altranais. Guidance to nurses and midwives on medication management. Dublin: An Bord Altranais, 2007.

2. Elliott M, Liu Y. The nine rights of medication administration: an overview. Br J Nurs 2010;19:300-5.

3. United Nations Department of Economic and Social Affairs Population Division. World population ageing 2013, 2013. ST/ESA/ SER.A/348.

4. United Nations Department of Economic and Social Affairs Population Division. World population prospects: the 2015 revision, key findings and advance tablets, 2015. Working Paper No. ESA/P/ WP.241.

5. World Health Organisation. World report on ageing and health, 2015.

6. Sabaté E. Adherence to long-term therapies: evidence for action. Geneva: World Health Organization, 2003.

7. Hajjar ER, Cafiero AC, Hanlon JT. Polypharmacy in elderly patients. Am J Geriatr Pharmacother 2007;5:345-51.

8. Stegemann S, Ecker F, Maio M, et al. Geriatric drug therapy: neglecting the inevitable majority. Ageing Res Rev 2010;9:384-98.

9. Topinková E, Baeyens JP, Michel JP, et al. Evidence-based strategies for the optimization of pharmacotherapy in older people. Drugs Aging 2012;29:477-94.

10. Aslam M, Vaezi MF. Dysphagia in the elderly. Gastroenterol Hepatol 2013;9:784-95. 
11. Chen PH, Golub JS, Hapner ER, et al. Prevalence of perceived dysphagia and quality-of-life impairment in a geriatric population. Dysphagia 2009;24:1-6.

12. Steele CM, Greenwood C, Ens I, et al. Mealtime difficulties in a home for the aged: not just dysphagia. Dysphagia 1997;12:43-50.

13. Hämmerlein A, Derendorf H, Lowenthal DT. Pharmacokinetic and pharmacodynamic changes in the elderly. Clinical implications. Clin Pharmacokinet 1998;35:49-64.

14. Mangoni AA, Jackson SH. Age-related changes in pharmacokinetics and pharmacodynamics: basic principles and practical applications. Br J Clin Pharmacol 2004;57:6-14.

15. Mercovich N, Kyle GJ, Naunton M. Safe to crush? A pilot study into solid dosage form modification in aged care. Australas $J$ Ageing 2014;33:180-4.

16. Caussin M, Mourier W, Philippe S, et al. [Crushing drugs in geriatric units: an "handicraft" practice with frequent errors which imposed recommendations]. Rev Med Interne 2012;33:546-51.

17. Fischbach MS, Gold JL, Lee M, et al. Pill-splitting in a long-term care facility. CMAJ 2001;164:785-6.

18. Mc Gillicuddy A, Kelly M, Sweeney C, et al. Modification of oral dosage forms for the older adult: An Irish prevalence study. Int $J$ Pharm 2016;510:386-93.

19. Haw C, Stubbs J. Covert administration of medication to older adults: a review of the literature and published studies. $J$ Psychiatr Ment Health Nurs 2010;17:761-8.

20. Kirkevold $\varnothing$, Engedal K. Concealment of drugs in food and beverages in nursing homes: cross sectional study. BMJ 2005;330:20.

21. National Institute for Health and Care Excellence. Medicines management in care homes, 2015.

22. Mental Welfare Commission for Scotland. Good practice guidecovert medication, 2013

23. Liabeuf S, Gras V, Moragny J, et al. Ulceration of the oral mucosa following direct contact with ferrous sulfate in elderly patients: a case report and a review of the French National Pharmacovigilance Database. Clin Interv Aging 2014;9:737-40.

24. Schier JG, Howland MA, Hoffman RS, et al. Fatality from administration of labetalol and crushed extended-release nifedipine. Ann Pharmacother 2003;37:1420-3.

25. Verrue $\mathrm{C}$, Mehuys E, Boussery K, et al. Tablet-splitting: a common yet not so innocent practice. J Adv Nurs 2011;67:26-32.

26. James $\mathrm{A}$. The legal and clinical implications of crushing tablet medication. Nurs Times 2004;100:28-9.

27. Wright $\mathrm{D}$, Chapman N, Foundling-Miah M, et al. Consensus guideline on the medication management of adults with swallowing difficulties. Berkhamsted: Medenium Group Publishing, 2006.

28. Griffith R, Davies R. Tablet crushing and the law: the implications for nursing. Prof Nurse 2003;19:41-2.

29. Paradiso LM, Roughead EE, Gilbert AL, et al. Crushing or altering medications: what's happening in residential aged-care facilities? Australas J Ageing 2002;21:123-7.

30. Braun V, Clarke V. Successful qualitative research: a practical guide for beginners: Sage, 2013

31. Kelly J, D'Cruz G, Wright D. A qualitative study of the problems surrounding medicine administration to patients with dysphagia. Dysphagia 2009;24:49-56.

32. Kelly J, D'Cruz G, Wright D. Patients with dysphagia: experiences of taking medication. J Adv Nurs 2010;66:82-91.

33. Barnes $L$, Cheek J, Nation RL, et al. Making sure the residents get their tablets: medication administration in care homes for older people. J Adv Nurs 2006:56:190-9.

34. Mc Gillicuddy A, Kelly M, Crean AM, et al. The knowledge, attitudes and beliefs of patients and their healthcare professionals around oral dosage form modification: A systematic review of the qualitative literature. Res Social Adm Pharm 2017;13.

35. Central Statistics Office Central Statistics Office. Census 2016 Small Area Population Statistics. http://census.cso.ie/sapmap/ (accessed 22 Aug 2017).

36. Marshall MN. Sampling for qualitative research. Fam Pract 1996;13:522-6.

37. Nursing Homes Ireland. Nursing Home Directory. http://www.nhi.ie/ directory.php (accessed 2 Feb 2016).

38. Cahill S, O'Caheny D. Specialist Care Units for People with Dementia in Ireland: A Guide for Family Caregivers and Health Service
Professionals. Dublin: The Dementia Services Information and Development Centre, St. James's Hospital, 2014.

39. Department of Health. Review of the nursing homes support scheme, a fair deal, 2015 http://health.gov.ie/wp-content/uploads/2015/07/ Review-of-Nursing-Homes-Support-Scheme.pdf

40. Bourdenet G, Giraud S, Artur M, et al. Impact of recommendations on crushing medications in geriatrics: from prescription to administration. Fundam Clin Pharmacol 2015;29:316-20.

41. Fodil M, Nghiem D, Colas M, et al. Assessment of clinical practices for crushing medication in geriatric units. J Nutr Health Aging 2017;21:904-8.

42. Francis $\mathrm{JJ}$, Johnston $\mathrm{M}$, Robertson $\mathrm{C}$, et al. What is an adequate sample size? Operationalising data saturation for theory-based interview studies. Psychol Health 2010;25:1229-45.

43. Braun V, Clarke V. Using thematic analysis in psychology. Qual Res Psychol 2006;3:77-101.

44. Landis JR, Koch GG. The measurement of observer agreement for categorical data. Biometrics 1977;33:159-74.

45. Tong A, Sainsbury P, Craig J. Consolidated criteria for reporting qualitative research (COREQ): a 32-item checklist for interviews and focus groups. Int J Qual Health Care 2007;19:349-57.

46. Serrano Santos JM, Poland F, Wright D, et al. Medicines administration for residents with dysphagia in care homes: A small scale observational study to improve practice. Int J Pharm 2016;512:416-21.

47. Wong IC, Basra N, Yeung VW, et al. Supply problems of unlicensed and off-label medicines after discharge. Arch Dis Child 2006;:91:686-8.

48. Mc Gillicuddy A, Crean AM, Sahm LJ. Older adults with difficulty swallowing oral medicines: a systematic review of the literature. Eur $J$ Clin Pharmacol 2016.72.

49. Hurst K. How much time do nurses spend at the bedside? Nurs Stand 2010;24:14

50. Zolnierek CD. An integrative review of knowing the patient. $J$ Nurs Scholarsh 2014;46:3-10.

51. Radwin LE. 'Knowing the patient': a review of research on an emerging concept. J Adv Nurs 1996;23:1142-6.

52. Suhonen R, Papastavrou E, Efstathiou G, et al. Patient satisfaction as an outcome of individualised nursing care. Scand J Caring Sci 2012;26:372-80.

53. Hendrich A, Chow MP, Skierczynski BA, et al. A 36-hospital time and motion study: how do medical-surgical nurses spend their time? Perm J 2008:12:25-34.

54. Strachan I, Greener M. Medication-related swallowing difficulties may be more common than we realise. Pharm Pract 2005;15:411-4.

55. Schiele JT, Quinzler R, Klimm H-D, et al. Difficulties swallowing solid oral dosage forms in a general practice population: prevalence, causes, and relationship to dosage forms. Eur J Clin Pharmacol 2013;69:937-48.

56. Greener M. Dealing with dysphagia. Geriatric Medicine 2008;38:539-42.

57. Arnet I, Hersberger KE. Misleading score-lines on tablets: facilitated intake or fractional dosing? Swiss Med Wkly 2010;140:105-10.

58. Food and Drug Administration. Guidance for Industry Tablet Scoring: Nomenclature, Labeling and Data for Evaluation. 2013 http://www.f da.gov/downloads/drugs/guidancecomplianceregulatoryinformation/ guidances/ucm269921.pdf

59. European Medicines Agency. Guideline on pharmaceutical development of medicines for paediatric use. 2013 http://www.ema. europa.eu/docs/en_GB/document_library/Scientific_guideline/2013/ 07/WC500147002.pdf

60. Messina R, Becker R, van Riet-Nales DA, et al. Results from a preliminary review of scientific evidence for appropriateness of preparations, dosage forms and other product design elements for older adult patients. Int J Pharm 2015;478:822-8.

61. Stegemann S, Gosch M, Breitkreutz J. Swallowing dysfunction and dysphagia is an unrecognized challenge for oral drug therapy. Int $J$ Pharm 2012;430:197-206.

62. Greenhalgh T, Snow R, Ryan S, et al. Six 'biases' against patients and carers in evidence-based medicine. BMC Med 2015;13:200.

63. Takase M. The relationship between the levels of nurses' competence and the length of their clinical experience: a tentative model for nursing competence development. J Clin Nurs 2013;22:1400-10. 